\title{
Bortezomib and belinostat inhibit renal cancer growth synergistically by causing ubiquitinated protein accumulation and endoplasmic reticulum stress
}

\author{
TAKAKO ASANO, AKINORI SATO, MAKOTO ISONO, KAZUKI OKUBO, KEIICHI ITO and TOMOHIKO ASANO \\ Department of Urology, National Defense Medical College, Tokorozawa, Saitama 359-8513, Japan
}

Received August 11, 2015; Accepted September 15, 2015

DOI: $10.3892 /$ br.2015.523

\begin{abstract}
There is no curative treatment for advanced renal cancer, and a novel treatment approach is urgently required. Inducing ubiquitinated protein accumulation and endoplasmic reticulum (ER) stress has recently emerged as a new approach in the treatment of malignancies. In the present study, we hypothesized that the histone deacetylase inhibitor belinostat would increase the amount of unfolded proteins in cells by inhibiting heat-shock protein (HSP) 90, and that the proteasome inhibitor bortezomib would inhibit their degradation by inhibiting the proteasome, thus causing ubiquitinated protein accumulation and ER stress synergistically. The combination of bortezomib and belinostat induced significant increases in apoptosis and inhibited renal cancer growth synergistically (combination indexes $<1$ ). The combination also suppressed colony formation significantly $(\mathrm{P}<0.05)$. As co-treatment with the pan-caspase inhibitor Z-VAD-FMK changed the number of Annexin V-positive cells, this combination-induced apoptosis was considered caspase dependent. Mechanistically, the combination synergistically caused ubiquitinated proteins to accumulate and induced ER stress, as evidenced by the increased expression of glucose-regulated protein 78 and HSP70. To the best of our knowledge, this is the first study demonstrating the beneficial combined effect of bortezomib and belinostat in renal cancer cells. The study provides a basis for clinical studies with the combination in patients with advanced renal cancer.
\end{abstract}

\section{Introduction}

There is no curative treatment for advanced renal cancer. Innovative treatments using tyrosine kinase inhibitors and inhibitors of the mammalian target of rapamycin have been tested, however, they were not curative (1-3). Development of a novel treatment approach is urgently required.

Correspondence to: Dr Akinori Sato, Department of Urology, National Defense Medical College, 3-2 Namiki, Tokorozawa, Saitama 359-8513, Japan

E-mail: zenpaku@ndmc.ac.jp

Key words: bortezomib, belinostat, endoplasmic reticulum stress, ubiquitinated protein, renal cancer
Inducing endoplasmic reticulum (ER) stress and ubiquitinated protein accumulation has emerged as a new approach for the treatment of malignancies (4). How effectively ER stress can be induced depends on how effectively unfolded proteins can be accumulated in the cell. Combining a proteasome inhibitor and histone deacetylase (HDAC) inhibitor is one efficient way to induce ER stress. HDAC inhibitors generally inhibit HDAC6, and ablation of HDAC6 induces hyperacetylation of heat-shock protein (HSP) 90, disrupting its chaperone function (5) and increasing unfolded proteins in the cell. These unfolded proteins, however, are normally degraded by the proteasome (6), so a proteasome inhibitor in combination with an HDAC inhibitor is expected to effectively accumulate unfolded proteins in the cell by inhibiting degradation of the unfolded proteins increased by the HDAC inhibitor. Our previous study revealed that the combinations of the proteasome inhibitor bortezomib and the HDAC inhibitor suberoylanilide hydroxamic acid (7) or panobinostat (8) induced ER stress and inhibited renal cancer growth synergistically. Combining bortezomib and HDAC inibitors would thus be a promising approach to causing ER stress in renal cancer cells.

Belinostat is a novel pandeacetylase inhibitor that has been clinically tested in patients with solid tumors, such as liver cancer (9), ovarian cancer (10), malignant pleural mesothelioma (11) and thymic epithelial tumors (12), as well as in patients with hematological malignancies $(13,14)$. Favorable antineoplastic effects of belinostat in combination with bortezomib have been reported (15-18), however, there has been no study evaluating the effect of the combination on renal cancer cells and investigating ER stress induction as a mechanism of action. Furthermore, belinostat is the only HDAC inhibitor that can be administered in multiple ways (i.e., intravenously or orally) (19) and may thus be easier to use than other HDAC inhibitors.

In the present study, the efficacy of the combination of bortezomib and belinostat was evaluated in renal cancer cells and its underlying mechanism of action was clarified, focusing on ER stress induction and ubiquitinated protein accumulation.

\section{Materials and methods}

Cell lines. Renal cancer cell lines (769-P, 786-O and $\mathrm{ACHN}$ ) were purchased from American Type Culture Collection (Rockville, MD, USA). Cells were grown in either 
RPMI or DMEM medium containing $10 \%$ fetal bovine serum and $0.3 \%$ penicillin-streptomycin (Invitrogen, Carlsbad, CA, USA).

Reagents. Belinostat and bortezomib were purchased from Selleck Chemicals (Houston, TX, USA) and LC Laboratories (Woburn, MA, USA), respectively. They were dissolved in dimethyl sulfoxide and stored at $-20^{\circ} \mathrm{C}$ until use. Z-VAD-FMK was obtained from Enzo Life Sciences (Farmingdale, NY, USA).

Cell viability assay. In total, $5 \times 10^{3}$ cells were seeded in a 96-well culture plate one day before treatment for $48 \mathrm{~h}$ with $0-2 \mu \mathrm{M}$ belinostat and either 0,5 , or $10 \mathrm{nM}$ bortezomib. Cell viability was subsequently assayed using the MTS assay (CellTiter 96 AQueous kit; Promega Madison, WI, USA) according to the manufacturer's protocol.

Colony formation assay. A total of 100 cells were seeded in 6-well plates one day before culture for $48 \mathrm{~h}$ in media containing $1 \mu \mathrm{M}$ belinostat and/or $10 \mathrm{nM}$ bortezomib. The cells were subsequently provided fresh media and allowed to grow for one week before being fixed with $100 \%$ methanol, stained with Giemsa's solution and counted.

Cell cycle analysis. In total, $1.5 \times 10^{5}$ cells were plated in 6-well culture plates one day before treatment for $48 \mathrm{~h}$ with $2 \mu \mathrm{M}$ belinostat and/or $10 \mathrm{nM}$ bortezomib, washed with phosphate-buffered saline and harvested by trypsinization. The cells were resuspended in citrate buffer and stained with propidium iodide according to the procedure used by Vindeløv et al (20), and were analyzed by flow cytometry.

Annexin V assay. A total of $1.5 \times 10^{5}$ cells were plated in 6-well culture plates one day before culture for $48 \mathrm{~h}$ in media containing $2 \mu \mathrm{M}$ belinostat and $10 \mathrm{nM}$ bortezomib with or without $40 \mu \mathrm{M}$ of the pancaspase inhibitor Z-VAD-FMK. The cells were subsequently stained with Annexin $\mathrm{V}$ according to the manufacturer's protocol (Beckman Coulter, Marseille, France). In total, 10,000 cells were counted and analyzed by flow cytometry.

Western blotting. Equal amounts of protein were subjected to SDS-PAGE and transferred to a nitrocellulose membrane. Following blocking with $5 \%$ skimmed milk, the membrane was incubated with a rabbit polyclonal antibody against glucose-regulated protein (GRP) 78 (1:200), a rabbit polyclonal antibody against ubiquitin (1:200) (Santa Cruz Biotechnology, Dallas, TX, USA), a mouse monoclonal antibody against actin $(1: 3,000)$ (Millipore, Billerica, MA, USA), a rabbit polyclonal antibody against HSP70 $(1: 1,000)$ and a rabbit polyclonal antibody against cleaved poly (ADP-ribose) polymerase (PARP) $(1: 1,000)$ (Cell Signaling Technology, Danvers, MA, USA). The samples were subsequently incubated with horseradish-peroxidase-tagged secondary antibodies (Bio-Rad, Hercules, CA, USA) and visualization was performed by chemiluminescence (ECL, Amersham, Piscataway, NJ, USA).

Statistical analysis. Evaluation of synergism was carried out using commercially available software (CalcuSyn, Biosoft, Cambridge, UK). The statistical significance of the observed
Table I. CI for the combination of bortezomib and belinostat.

\begin{tabular}{lccc}
\hline & & \multicolumn{2}{c}{ Bortezomib, $\mathrm{nM}$} \\
\cline { 3 - 4 } Cell line & Belinostat, $\mu \mathrm{M}$ & 5 & 10 \\
\hline $769-\mathrm{P}$ & 0.5 & 1.029 & $0.529^{\mathrm{a}}$ \\
& 1 & $0.685^{\mathrm{a}}$ & $0.405^{\mathrm{a}}$ \\
& 2 & $0.579^{\mathrm{a}}$ & $0.286^{\mathrm{a}}$ \\
$786-\mathrm{O}$ & 0.5 & 1.287 & $0.949^{\mathrm{a}}$ \\
& 1 & 1.084 & 1.142 \\
& 2 & 1.099 & $0.813^{\mathrm{a}}$ \\
ACHN & 0.5 & $0.075^{\mathrm{a}}$ & $0.093^{\mathrm{a}}$ \\
& 1 & $0.115^{\mathrm{a}}$ & $0.127^{\mathrm{a}}$ \\
& 2 & $0.073^{\mathrm{a}}$ & $0.129^{\mathrm{a}}$ \\
\end{tabular}

${ }^{\mathrm{a}} \mathrm{CI}<1$ indicates synergism. $\mathrm{CI}$, combination indexes.

differences in the colony formation assay was evaluated using the Mann-Whitney U test (StatView software, SAS Institute, Cary, NC, USA). P $<0.05$ was considered to indicate a statistically significant difference.

\section{Results}

Bortezomib and belinostat synergistically inhibit renal cancer growth. The combined effect of bortezomib and belinostat were first examined on cell viability. Bortezomib and belinostat each inhibited the growth of renal cancer cells in a dose-dependent manner and the combination inhibited their growth more than treatment with the agents individually (Fig. 1A and B). Subsequently, the interaction of these two agents was evaluated using the Chou-Talalay method to calculate the combination indexes. Indexes $<1$ showed that their interaction was synergistic under a number of the treatment conditions (Table I). Whether the combination of bortezomib and belinostat interferes with colony formation by renal cancer cells was subsequently investigated. The combination suppressed colony formation significantly more than $10 \mathrm{nM}$ bortezomib or $1 \mu \mathrm{M}$ belinostat alone (Fig. 1C). Therefore, the combination of bortezomib and belinostat was shown to inhibit renal cancer growth effectively.

Bortezomib and belinostat induce apoptosis. Cell cycle analysis and the Annexin $\mathrm{V}$ assay were used to observe whether the combination of bortezomib and belinostat induced apoptosis. Cell cycle analysis (Fig. 2A) showed that the combination of $10 \mathrm{nM}$ bortezomib and $2 \mu \mathrm{M}$ belinostat synergistically increased the number of the cells in the sub- $G_{1}$ fraction $(\leq 98.3 \%)$. The combination also markedly increased Annexin V-FITC fluorescence intensity (Fig. 2B) and increased the expression of cleaved PARP (Fig. 2C). Thus, the combination was shown to induce apoptosis. Whether the combination-induced apoptosis is associated with caspase activation was also examined. As co-treatment with a $40 \mu \mathrm{M}$ concentration of the pan-caspase inhibitor Z-VAD-FMK changed the number of Annexin V-positive cells (reduced in 769-P cells and increased in 786-O and ACHN cells), the apoptosis was considered, at least in part, caspase dependent (Fig. 2B). 
A
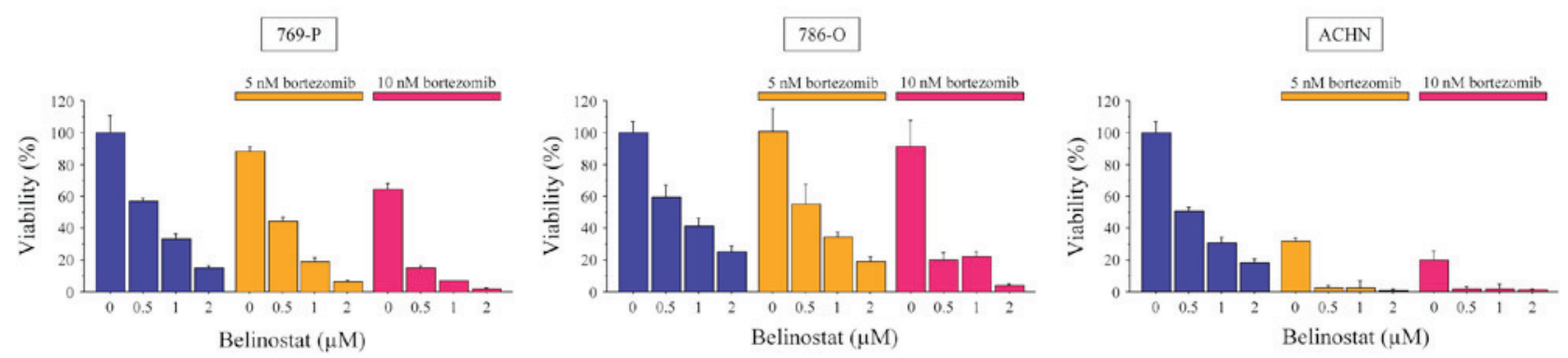

B

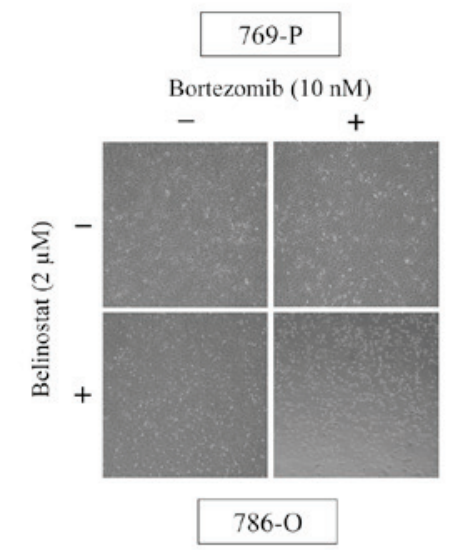

Bortezomib (10 nM)

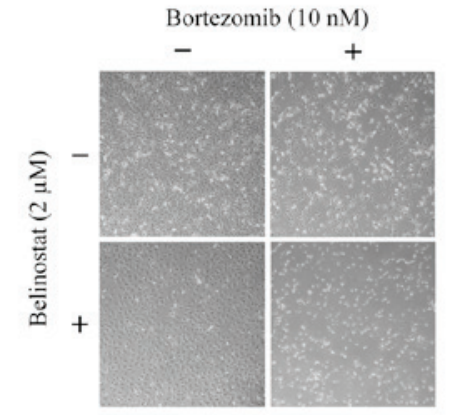

C
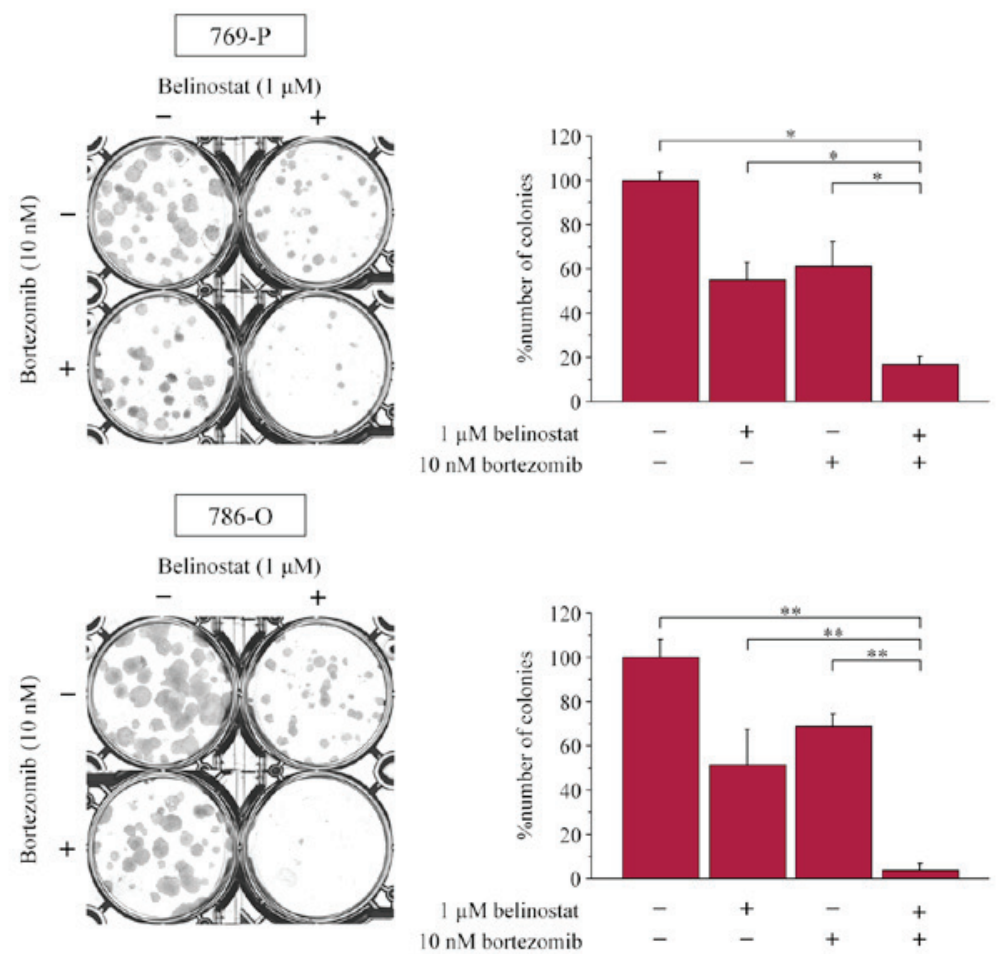

Figure 1. Bortezomib and belinostat kill renal cancer cells. (A) MTS assay $(n=6$, mean \pm SD). Cells were treated for $48 \mathrm{~h}$ with the indicated concentrations of bortezomib and/or belinostat. (B) Photomicrographs of 769-P and 786-O cells treated with $10 \mathrm{nM}$ bortezomib and/or $2 \mu \mathrm{M}$ belinostat. The majority of the cells treated with the combination are floating. Original magnification, $x 40$. (C) Colony formation assay. A total of 100 cells were plated in 6-well plates one day before culture for $48 \mathrm{~h}$ in media containing $10 \mathrm{nM}$ bortezomib and/or $1 \mu \mathrm{M}$ belinostat. The cells were subsequently provided with fresh media and allowed to grow for $1-2$ weeks $\left(\mathrm{n}=3\right.$, mean $\left.\pm \mathrm{SD},{ }^{*} \mathrm{P}=0.0495,{ }^{* * *} \mathrm{P}=0.0463\right)$. $\mathrm{SD}$, standard deviation.

Bortezomib and belinostat cause ubiquitinated protein accumulation and ER stress. Our hypothesis was that the combination of bortezomib and belinostat would increase ubiquitinated proteins in the cell and induce ER stress cooperatively, as belinostat would increase the amount of unfolded proteins and bortezomib would inhibit their degradation by inhibiting the proteasome. As expected, the combination synergistically caused ubiquitinated proteins to accumulate (Fig. 3). In all three cell lines, no accumulation of ubiquitinated protein was induced by $2 \mu \mathrm{M}$ belinostat alone; however, in combination with $10 \mathrm{nM}$ bortezomib, marked accumulation of ubiquitinated protein was induced. By contrast, $10 \mathrm{nM}$ bortezomib caused small (in 769-P and ACHN cells) and moderate (in 786 cells) ubiquitinated protein accumulation that was markedly enhanced by belinostat. The combination also increased the expression of the ER stress markers GRP78 and HSP70, confirming that the combination actually caused ubiquitinated unfolded protein accumulation and thereby induced ER stress.

\section{Discussion}

There is no curative therapy for advanced renal cancer, and a novel treatment approach is urgently required. Inducing ubiquitinated protein accumulation and ER stress is a new approach to the treatment of malignancies (4) and our laboratory has been investigating ways to kill urological cancer cells using clinically feasible drugs to induce ER stress. To the best of our knowledge, for the first time the favorable combined effect of the proteasome inhibitor bortezomib and the HDAC inhibitor belinostat in renal cancer was revealed in the present study.

Combining a proteasome inhibitor and HDAC inhibitor is one effective way to induce ER stress, as HDAC inhibitors are considered to increase unfolded proteins by suppressing HSP90 function (5) and proteasome inhibitors inhibit the degradation of those unfolded proteins. This strategy has been shown to be effective in killing renal cancer cells $(7,8)$.

The present analysis using the Chou-Talalay method showed that the combination of bortezomib and belinostat inhibited 
A

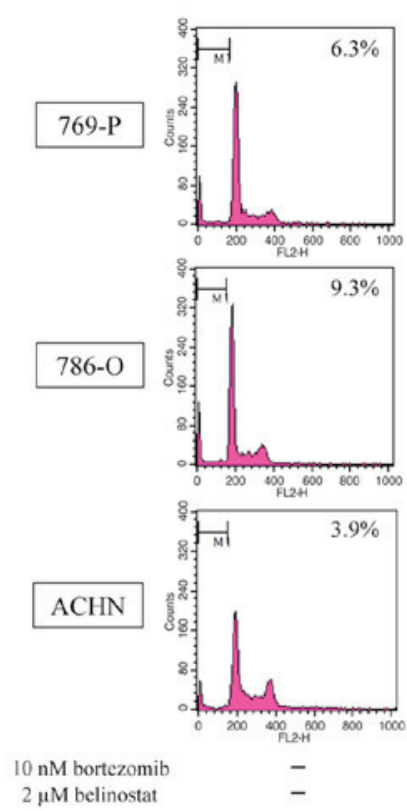

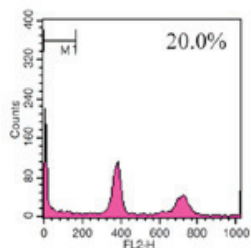
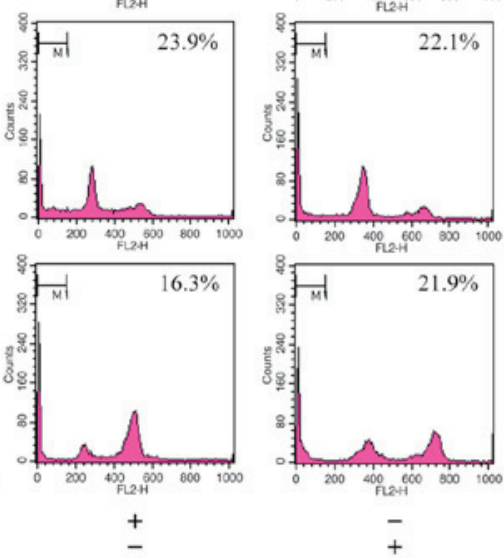
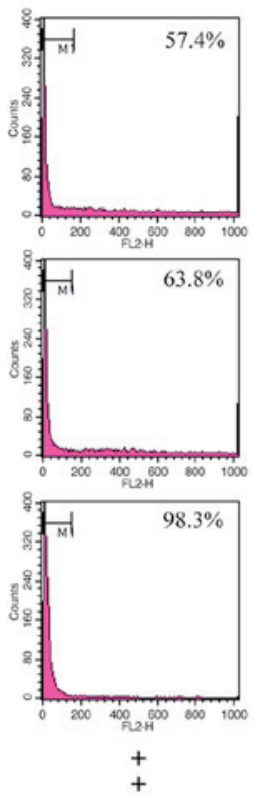

B

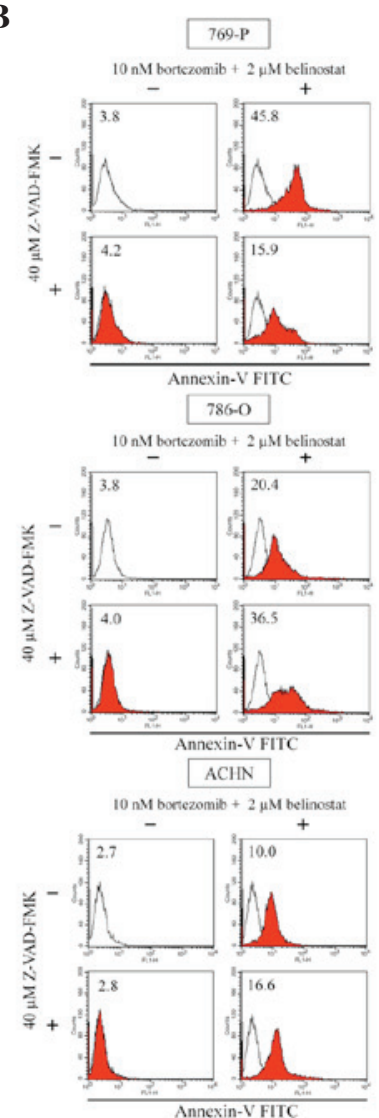

Figure 2. Bortezomib and belinostat induce apoptosis. (A) Cell cycle analysis. Cells were cultured for $48 \mathrm{~h}$ in medium containing $10 \mathrm{nM}$ bortezomib and/or $2 \mu \mathrm{M}$ belinostat. In total, 10,000 cells were counted and changes in the cell cycle were evaluated using flow cytometry. The inset in each graph is the percentage of cells in the sub- $\mathrm{G}_{1}$ fraction. (B) Annexin $\mathrm{V}$ assay. Cells were cultured for $48 \mathrm{~h}$ in medium containing $10 \mathrm{nM}$ bortezomib and $2 \mu \mathrm{M}$ belinostat with or without $40 \mu \mathrm{M}$ pancaspase inhibitor Z-VAD-FMK. In total, 10,000 cells were analyzed by the Annexin V assay using flow cytometry. The inset in each graph is the Annexin V-FITC fluorescence intensity. (C) Western blot analysis results. Cells were treated with $1-2 \mu \mathrm{M}$ belinostat with or without $10 \mathrm{nM}$ bortezomib for $48 \mathrm{~h}$. Actin was used for the loading control. Representative blots are shown.

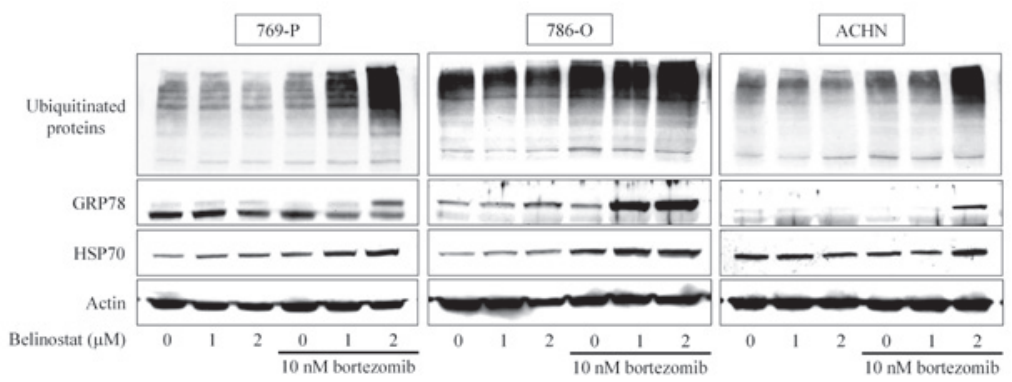

Figure 3. Bortezomib and belinostat cause ubiquitinated protein accumulation and endoplasmic reticulum stress. Cells were treated for $48 \mathrm{~h}$ with 1-2 $\mu \mathrm{M}$ belinostat with or without $10 \mathrm{nM}$ bortezomib and the expression of ubiquitinated proteins, GRP78 and HSP70 were evaluated using western blotting. Actin was used for the loading control. Representative blots are shown. GRP, glucose-regulated protein; HSP, heat-shock protein.

renal cancer growth synergistically. It caused ubiquitinated protein accumulation and ER stress cooperatively: $2 \mu \mathrm{M}$ belinostat induced marked accumulation of ubiquitinated protein only when it was combined with $10 \mathrm{nM}$ bortezomib. Therefore, the increased amount of unfolded proteins due to $2 \mu \mathrm{M}$ belinostat alone did not overwhelm the protein degrading ability of the proteasome; i.e., they were rapidly degraded by the proteasome and therefore did not accumulate. By contrast, in 769-P and ACHN cells, $10 \mathrm{nM}$ bortezomib alone caused only small ubiquitinated protein accumulation; however, in combination with belinostat it caused marked ubiquitinated protein accumulation. Thus, in these cell lines the amount of unfolded protein must be increased for effective ubiquitinated protein accumulation, even though the proteasome is suppressed. Simultaneous inhibition of HSP90 and proteasome function is therefore of importance in causing unfolded proteins to accumulate and inducing enough ER stress to kill cancer cells. As the proteasomes in renal cancer cells are overexpressed (21), this proteasome inhibition is considered particularly important when killing renal cancer cells by inducing ER stress. 
As indicated by the cell cycle analysis, Annexin V assay and western blot analysis detecting cleaved PARP, the combination induced apoptosis effectively. This apoptosis is considered to be associated with caspase activation as the pancaspase inhibitor Z-VAD-FMK affected the number of Annexin V-positive cells caused by the combination. Notably, the effect of Z-VAD-FMK differed among the cell lines: Decreasing the number of Annexin V-positive cells in 769-P cells and increasing it in 786-O and ACHN cells. Therefore, in 786-O and ACHN cells the inhibition of caspase paradoxically promoted apoptosis. A similar phenomenon was reported by Liu et al (22) who showed that Z-VAD-FMK sensitized neutrophils to tumor necrosis factor- $\alpha$-induced apoptosis using the Annexin V assay. The study hypothesized that the caspase-dependent pathway normally suppresses or supersedes caspase-independent mechanisms; however, understanding the exact mechanism of this paradoxical phenomenon will require further study.

One limitation of the present study is that suppression of HSP90 function by belinostat was not shown. Belinostat individually induced histone acetylation at the indicated concentrations, so it is evident that belinostat suppressed the function of HDACs, and we hypothesized that belinostat would therefore cause HSP90 acetylation, leading to its functional suppression. However, formal functional evaluation of HSP90 under belinostat treatment would be the subsequent step in clarifying the actual mechanism of action of belinostat in this combination therapy.

In conclusion, the bortezomib-belinostat combination induced ubiquitinated protein accumulation and ER stress, and killed renal cancer cells synergistically. To the best of our knowledge, this is the first study to demonstrate the favorable combined effect of bortezomib and belinostat in renal cancer cells. As this combination kills renal cancer cells in a way that is completely different from the non-curative treatments currently used, we hypothesize that the present study, along with our previous studies, provides a framework for testing combinations of proteasome inhibitors and HDAC inhibitors in patients with advanced renal cancer.

\section{References}

1. Escudier B, Eisen T, Stadler WM, Szczylik C, Oudard S, Siebels M, Negrier S, Chevreau C, Solska E, Desai AA, et al; TARGET Study Group: Sorafenib in advanced clear-cell renal-cell carcinoma. N Engl J Med 356: 125-134, 2007.

2. Motzer RJ, Rini BI, Bukowski RM, Curti BD, George DJ, Hudes GR, Redman BG, Margolin KA, Merchan JR, Wilding G, et al: Sunitinib in patients with metastatic renal cell carcinoma. JAMA 295: 2516-2524, 2006.

3. Motzer RJ, Escudier B, Oudard S, Hutson TE, Porta C, Bracarda S, Grünwald V, Thompson JA, Figlin RA, Hollaender N, et al; RECORD-1 Study Group: Phase 3 trial of everolimus for metastatic renal cell carcinoma: Final results and analysis of prognostic factors. Cancer 116: 4256-4265, 2010.

4. Liu Y and Ye Y: Proteostasis regulation at the endoplasmic reticulum: A new perturbation site for targeted cancer therapy. Cell Res 21: 867-883, 2011.

5. Bali P, Pranpat M, Bradner J, Balasis M, Fiskus W, Guo F, Rocha K, Kumaraswamy S, Boyapalle S, Atadja P, et al: Inhibition of histone deacetylase 6 acetylates and disrupts the chaperone function of heat shock protein 90: A novel basis for antileukemia activity of histone deacetylase inhibitors. J Biol Chem 280: 26729-26734, 2005.
6. Naujokat $\mathrm{C}$ and Hoffmann S: Role and function of the $26 \mathrm{~S}$ proteasome in proliferation and apoptosis. Lab Invest 82: 965-980, 2002.

7. Sato A, Asano T, Ito K, Sumitomo M and Asano T: Suberoylanilide hydroxamic acid (SAHA) combined with bortezomib inhibits renal cancer growth by enhancing histone acetylation and protein ubiquitination synergistically. BJU Int 109: 1258-1268, 2012.

8. Sato A, Asano T, Isono M, Ito K and Asano T: Panobinostat synergizes with bortezomib to induce endoplasmic reticulum stress and ubiquitinated protein accumulation in renal cancer cells. BMC Urol 14: 71, 2014.

9. Yeo W, Chung HC, Chan SL, Wang LZ, Lim R, Picus J, Boyer M, Mo FK, Koh J, Rha SY, et al: Epigenetic therapy using belinostat for patients with unresectable hepatocellular carcinoma: A multicenter phase I/II study with biomarker and pharmacokinetic analysis of tumors from patients in the Mayo Phase II Consortium and the Cancer Therapeutics Research Group. J Clin Oncol 30: 3361-3367, 2012.

10. Mackay HJ, Hirte H, Colgan T, Covens A, MacAlpine K, Grenci P, Wang L, Mason J, Pham PA, Tsao MS, et al: Phase II trial of the histone deacetylase inhibitor belinostat in women with platinum resistant epithelial ovarian cancer and micropapillary (LMP) ovarian tumours. Eur J Cancer 46: 1573-1579, 2010.

11. Ramalingam SS, Belani CP, Ruel C, Frankel P, Gitlitz B, Koczywas M, Espinoza-Delgado I and Gandara D: Phase II study of belinostat (PXD101), a histone deacetylase inhibitor, for second line therapy of advanced malignant pleural mesothelioma. J Thorac Oncol 4: 97-101, 2009.

12. GiacconeG,RajanA,BermanA,KellyRJ,SzaboE,Lopez-ChavezA, Trepel J, Lee MJ, Cao L, Espinoza-Delgado I, et al: Phase II study of belinostat in patients with recurrent or refractory advanced thymic epithelial tumors. J Clin Oncol 29: 2052-2059, 2011.

13. Cashen A, Juckett M, Jumonville A, Litzow M, Flynn PJ, Eckardt J, LaPlant B, Laumann K, Erlichman C and DiPersio J: Phase II study of the histone deacetylase inhibitor belinostat (PXD101) for the treatment of myelodysplastic syndrome (MDS). Ann Hematol 91: 33-38, 2012.

14. Gimsing P, Hansen M, Knudsen LM, Knoblauch P, Christensen IJ, Ooi CE and Buhl-Jensen P: A phase I clinical trial of the histone deacetylase inhibitor belinostat in patients with advanced hematological neoplasia. Eur J Haematol 81: $170-176,2008$

15. Paoluzzi L, Scotto L, Marchi E, Zain J, Seshan VE and O'Connor OA: Romidepsin and belinostat synergize the antineoplastic effect of bortezomib in mantle cell lymphoma. Clin Cancer Res 16: 554-565, 2010.

16. Spratlin JL, Pitts TM, Kulikowski GN, Morelli MP, Tentler JJ, Serkova NJ and Eckhardt SG: Synergistic activity of histone deacetylase and proteasome inhibition against pancreatic and hepatocellular cancer cell lines. Anticancer Res 31: 1093-1103, 2011.

17. Dai Y, Chen S, Wang L, Pei XY, Kramer LB, Dent P and Grant S: Bortezomib interacts synergistically with belinostat in human acute myeloid leukaemia and acute lymphoblastic leukaemia cells in association with perturbations in NF- $\kappa \mathrm{B}$ and Bim. Br J Haematol 153: 222-235, 2011.

18. Feng R, Oton A, Mapara MY, Anderson G, Belani C and Lentzsch S: The histone deacetylase inhibitor, PXD101, potentiates bortezomib-induced anti-multiple myeloma effect by induction of oxidative stress and DNA damage. Br J Haematol 139: 385-397, 2007.

19. Lemoine $\mathbf{M}$ and Younes A: Histone deacetylase inhibitors in the treatment of lymphoma. Discov Med 10: 462-470, 2010.

20. Vindel $\varnothing v$ LL, Christensen IJ and Nissen NI: A detergent-trypsin method for the preparation of nuclei for flow cytometric DNA analysis. Cytometry 3: 323-327, 1983.

21. Kanayama H, Tanaka K, Aki M, Kagawa S, Miyaji H, Satoh M, Okada F, Sato S, Shimbara N and Ichihara A: Changes in expressions of proteasome and ubiquitin genes in human renal cancer cells. Cancer Res 51: 6677-6685, 1991.

22. Liu CY, Takemasa A, Liles WC, Goodman RB, Jonas M, Rosen H, Chi E, Winn RK, Harlan JM and Chuang PI: Broad-spectrum caspase inhibition paradoxically augments cell death in TNF-alpha -stimulated neutrophils. Blood 101: 295-304, 2003. 